\title{
Österreichs Sozialstaat im Vergleich internationaler Makrodaten
}

\section{Herbert Obinger}

Zentrum für Sozialpolitik, Universität Bremen, Deutschland

E-Mail: herbert.obinger@uni-bremen.de

\section{Zusammenfassung}

Dieser Beitrag vermisst anhand von über 30 Makroindikatoren das Profil und die Performanz des österreichischen Sozialstaates im internationalen Vergleich. Neben Ausgabenniveaus und Finanzierungsstruktur werden die Generosität und der Inklusionsgrad ausgewählter Sicherungssysteme sowie die Performanz in den Bereichen Einkommensverteilung und Beschäftigung in den Blick genommen. Der Vergleich zeigt, dass Österreichs Sozialstaat trotz ausgeprägter Bismarckischer Strukturmerkmale eine deutlich überdurchschnittliche Leistungsfähigkeit aufweist.

\section{Schlüsselwörter}

Österreich, Bismarcksche Wohlfahrtsstaaten, internationaler Vergleich, Makroindikatoren

\section{The Austrian welfare state in international comparison of macro-indicators}

\begin{abstract}
Based on more than 30 macro-indicators this article gauges the profile and performance of the Austrian welfare state in international comparison. In addition to social spending levels and patterns of financing, this paper sheds light on the generosity of welfare benefits and the coverage of selected welfare programs and examines Austria's performance in terms of income distribution and employment. The comparison demonstrates that the Austrian welfare state, despite its pronounced Bismarckian make-up, shows a very favourable performance.
\end{abstract}

\section{Keywords}

Austria, Bismarckian welfare states, international comparison, macro-indicators

The author has declared that no competing interests exist. 


\section{Einleitung}

Österreich ist eine der Pioniernationen staatlicher Sozialpolitik (Schmidt 2005) und gilt als Prototyp des kontinentaleuropäischen Sozialstaatsmodells (Esping-Andersen I990; Unger/Heitzmann 2003; Heitzmann/Österle 2008; Obinger/Tálos 20ı0; Tálos 2005; 2013). Diesem Sozialstaatstyp eilt jedoch ein schlechter Ruf voraus. Bismarckische Wohlfahrtsstaaten gelten in der Literatur als notorisch reformresistent, transferlastig, überreguliert, statusreproduzierend, beschäftigungsfeindlich, insiderfreundlich, genderantiquiert und insgesamt den nordischen Ländern hinsichtlich ihrer arbeitsmarkt- und sozialpolitischen Leistungsfähigkeit deutlich unterlegen (vgl. Esping-Andersen 1996; Palier 20I0; Palier/Thelen 20I0; Emmenegger et al. 20I2). Immerhin wird ihnen aber ein besserer Sozialschutz als den liberalen Wohlfahrtsstaaten der „Neuen Welt" attestiert.

Inwieweit entspricht aber das Profil und die Performanz des österreichischen Sozialstaates diesen Diagnosen? Was sind seine spezifischen Besonderheiten, Stärken und Schwächen und inwieweit trifft die gängige Verortung zwischen dem nordischen und liberalen Sozialstaatsmodell zu? Dieser Beitrag versucht diese Fragen aus einer vergleichenden Makroperspektive zu beantworten. Während für Deutschland einschlägige Untersuchungen bereits vorliegen (Alber 1998; Schmidt 20I2; Obinger 20I4), fehlt bislang ein solcher Vergleich für Österreich. Anhand von 33 Makroindikatoren sollen das Profil und die Leistungsfähigkeit des österreichischen Sozialstaates im Vergleich mit 22 OECD Ländern ${ }^{1}$ in drei Dimensionen vermessen werden. Die erste Dimension umfasst die gesamten und programmspezifischen Ausgaben für soziale Sicherung sowie die Art ihrer Finanzierung. Die zweite Dimension fokussiert auf den Policy-Output, nämlich die Generosität der Sozialleistungen, die Leistungsart, den erfassten Personenkreis und die Regulierungsdichte des Arbeitsmarktes. Schließlich werden die Auswirkungen (Outcomes) sozialstaatlicher Politik in den Bereichen Beschäftigung und Einkommensverteilung in den Blick genommen. Diese drei an die Policyforschung angelehnten Analysedimensionen strukturieren auch den Inhalt dieses Beitrags.

Der Vergleich erfolgt zu zwei Zeitpunkten (I980 und ca. 2OII) und umspannt damit einen Zeitraum, der in der gesamten OECD-Welt durch massive Veränderungen der sozio-ökonomischen und politischen Rahmenbedingungen und einen dadurch angeleiteten Umbau der sozialen Sicherungssysteme geprägt war (Gilbert 2002; Hemerijck 20I3; Obinger/Starke 20I5). Mit dieser intertemporalen Betrachtung können sowohl internationale sozialpolitische

I Australien, Belgien, Kanada, Dänemark, Finnland, Frankreich, Deutschland, Griechenland, Island, Irland, Italien, Japan, Luxemburg, Niederlande, Neuseeland, Norwegen, Portugal, Spanien, Schweden, Schweiz, Großbritannien, USA.
Entwicklungstrends als auch etwaige Positions- und Profilverschiebungen des österreichischen Sozialstaates im Zeitverlauf dokumentiert werden.

Die Auswahl der Indikatoren folgt zwei Gesichtspunkten. Zum einen handelt es sich bei den Outputund Outcomeindikatoren um Standardvariablen der Sozialstaatskomparatistik, wie sie etwa von der skandinavischen Machtressourcentheorie als wohl einflussreichster Theorieschule der vergleichenden Wohlfahrtsstaatsforschung vorgeschlagen werden. Sie reflektieren zentrale Funktionen des Wohlfahrtsstaates, nämlich die Gewährleistung von Einkommensersatz und sozialen Dienstleistungen im Risikofall bzw. in bestimmten Lebenslagen, und messen seinen Beitrag zur Armutsvermeidung und Korrektur von Marktergebnissen. Zudem werden die zur Erfüllung dieser Aufgaben bereitgestellten finanziellen Aufwendungen berücksichtigt. Zum anderen ist die Wahl der Indikatoren der Datenverfügbarkeit im Quer- und Längsschnitt geschuldet.

Zur Vermessung der Positionierung Österreichs im internationalen Vergleich werden hauptsächlich Boxplots (Kastenabbildungen) verwendet. Boxplots fassen zentrale Eigenschaften einer Verteilung wie Streuungs- und Lageparameter visuell kompakt zusammen und sind wie folgt $\mathrm{zu}$ interpretieren: Die Länge der Box beschreibt den so genannten Interquartilbereich, der die mittleren 50\% der Beobachtungswerte enthält. Das obere Ende der Box ist das obere Quartil, d.h. 75\% der Werte sind kleiner oder gleich groß wie dieser Wert. Das untere Ende der Box ist das untere Quartil, d.h. 25\% der Werte sind kleiner oder gleich groß ${ }^{2}$. Die fettgedruckte horizontale Linie innerhalb der Box ist der Median, der die Verteilung in zwei gleich große Gruppen teilt: 50\% der Beobachtungen liegen daher über diesem Wert, 50\% darunter. Die Lage des Median innerhalb der Box informiert darüber hinaus über die Schiefe der Verteilung. Befindet sich der Median genau in der Mitte der Box, dann sind die Beobachtungswerte symmetrisch verteilt. Liegt er am oberen Ende der Box ist die Verteilung linksschief, befindet er sich am unteren Ende, dann ist die Verteilung rechtsschief. Am oberen und unteren Ende der Box befinden sich die so genannten Whiskers oder Antennen. Sie zeigen den größten und kleinsten Wert der Verteilung an, sofern es sich um keine Ausreißer handelt. Statistische Ausreißer sind Werte, die mehr als I,5 Boxlängen von der Box entfernt platziert sind, und werden mit einem Kreis ausgewiesen. Gibt es keine Ausreißer, dann entspricht die Differenz zwischen

2 Die Differenz zwischen dem oberen und unteren Quartil (Q75Q25) ist der erwähnte Interquartilsabstand und ist identisch mit der Länge der Box. Die Boxlänge informiert daher über die Streuung der mittleren 50\% der Beobachtungswerte. 
den Enden der Whiskers (d.h. die Differenz zwischen dem Maximum- und Minimumwert) der Spannweite der Verteilung.

Vertraut man auf das Urteil der Fachwelt, so müsste Österreich als kontinentaleuropäischer Sozialversicherungsstaat einen "mittleren Weg" zwischen dem nordischen und angelsächsischen Modell beschreiten, wie es etwa der Bundesrepublik Deutschland - dem Archetyp des kontinentalen Sozialstaatsmodells - bescheinigt wird (Alber 1998; Schmidt 2012). Demnach müsste Österreich überproportional häufig im Interquartilbereich platziert sein. Dieser Beitrag zeigt, dass dies nicht der Fall ist. Österreichs Sozialstaat weist zwar im Hinblick auf zentrale Strukturmerkmale wie Finanzierungsform und Leistungsart ausgeprägte Bismarcksche Züge auf, ist aber im Hinblick auf zentrale Outcomes, die aus Sicht der Machtressourcentheorie zu den Kernaufgaben des Sozialstaates gehören (vgl. Korpi/Palme 2003), den nordischen Sozialstaaten fast ebenbürtig und vereinzelt sogar überlegen. Dessen ungeachtet gibt es Problemzonen. Diese werden sichtbar, wenn die Sozialstaatsarchitektur auf ihre Zukunftsfestigkeit überprüft wird. Dieser Aspekt wird im abschließenden Fazit diskutiert.

\section{Ausgaben und Finanzierung}

Die öffentlichen Bruttosozialausgaben bildeten über Jahrzehnte hinweg die zentrale abhängige Variable in der vergleichenden Sozialstaatsforschung. Weniger Berücksichtigung fand hingegen die Finanzierungsseite und erst seit Kurzem wird aus vergleichender Perspektive der Besteuerung von Sozialtransfers bzw. der steuerlichen Begünstigung privater und betrieblicher Vorsorge mehr Aufmerksamkeit gewidmet (Adema et al. 20II).

\section{Sozialausgaben}

Ungeachtet der berechtigten Kritik an ausgabenbasierten Sozialpolitikanalysen (Esping-Andersen 1990, 27) zeigen neuere Untersuchungen auf Basis einer massiv verbesserten Datenlage, dass die Höhe und Struktur der Sozialausgaben nicht nur stark mit alternativen Sozialstaatsindikatoren (z.B. dem Dekommodifizierungsgrad) korrelieren, sondern auch die verschiedenen Wohlfahrtsstaatstypen adäquat abbilden (Castles 2009; Obinger/Wagschal 20I0).

Abbildung I stellt die öffentlichen und privaten Ausgaben für soziale Sicherung dar. In der linken oberen Abbildung sind die öffentlichen Bruttosozialausgaben in Relation zum Bruttoinlandsprodukt (BIP), d.h. der klassische Sozialstaatsindikator, dargestellt. Daraus ist zunächst ersichtlich, dass die Sozialausgaben in der OECD-Welt seit 1980 deutlich gestiegen sind, wobei die jüngste Wirtschaftskrise mit einem weiteren Ausgaben- schub einherging ${ }^{3}$. Österreich liegt sowohl 1980 als auch 2012 deutlich über dem internationalen Durchschnitt. Blickt man auf das relative Gewicht des Sozialstaates, d.h. den Anteil der öffentlichen Sozialausgaben an den gesamten Ausgaben der öffentlichen Hand, dann ist ebenfalls ein Wachstum des Sozialstaats seit $199 \mathrm{I}^{4} \mathrm{zu}$ beobachten (rechte obere Abbildung). Besonders kräftig war dieser Zuwachs in Österreich, das nun zusammen mit Deutschland im internationalen Vergleich eine Spitzenposition einnimmt. Ca. 55 Prozent aller öffentlichen Ausgaben waren 2009 im Sozialbereich gebunden, während alle anderen Politikfelder (z.B. Wirtschaft, Bildung, Innere Sicherheit, Verteidigung, Verwaltung, Umwelt, Schuldendienst) um die verbleibenden Ausgaben konkurrierten.

Konträr zum internationalen Trend haben sich die privaten Pflichtleistungen entwickelt, die in Österreich gesunken sind und sich nun im Interquartilbereich befinden ${ }^{5}$ (linke untere Abbildung). Ebenfalls nur leicht überdurchschnittlich hoch sind die gesamten Nettosozialausgaben (rechte untere Abbildung), die neben sozialpolitisch motivierten Steuervergünstigungen auch die privaten Sozialausgaben berücksichtigen und die öffentlichen und privaten Sozialtransfers um die direkten und indirekten Steuer- und Abgabenlasten bereinigen (vgl. Adema et al. 20II). ${ }^{6}$ Die Nettosozialausgabenquote ist daher ein Maß dafür, wie viele Ressourcen in Relation zur Wirtschaftsleistung eines Landes für sozialpolitische Zwecke netto zur Verfügung stehen.

Die Social Expenditure Database der OECD liefert auch Daten zu den programmspezifischen Bruttosozialausgaben. Abbildung 2 zeigt die öffentlichen Ausgaben für die vier größten Einzelprogramme des Sozialstaates. Vor dem Hintergrund des Anstiegs der Bruttosozialleistungsquote ist es zunächst wenig überraschend, dass auch die programmspezifischen Aufwendungen in der OECD-Welt seit 1980 erheblich angestiegen sind. Österreich liegt mit Ausnahme der Ausgaben für Familienpolitik, die leicht gesunken sind, im internationalen Trend. Auffällig sind besonders die weit überdurchschnittlichen Ausgaben für die Alterssicherung. Hier nahm Österreich 1980 die Spitzenposition ein und wurde 2009 nur noch von Italien übertroffen. Daran ändert sich auch nichts, wenn die Hinterbliebenenversorgung berücksichtigt wird (nicht dargestellt). Bei den anderen drei Programmen liegt Österreich hingegen im Mittelfeld. Die Gesundheitsausgaben, die gesamten Fa-

3 Der Anstieg der Sozialausgabenquote im Zuge der Finanzkrise resultierte einerseits aus einem sinkenden BIP, andererseits aus der rezessionsbedingt höheren Nachfrage nach Sozialleistungen.

4 Erst ab diesem Zeitraum liegen Daten für mehrere Länder vor.

5 Hier handelt es sich um Leistungen, die gesetzlich vorgeschrieben, aber von privaten Akteuren finanziert werden.

6 Dieser von OECD-Experten berechnete Indikator ist erst seit wenigen Jahren verfügbar, sodass kein intertemporaler Vergleich möglich ist. 
Abbildung 1: Aggregierte Sozialausgaben

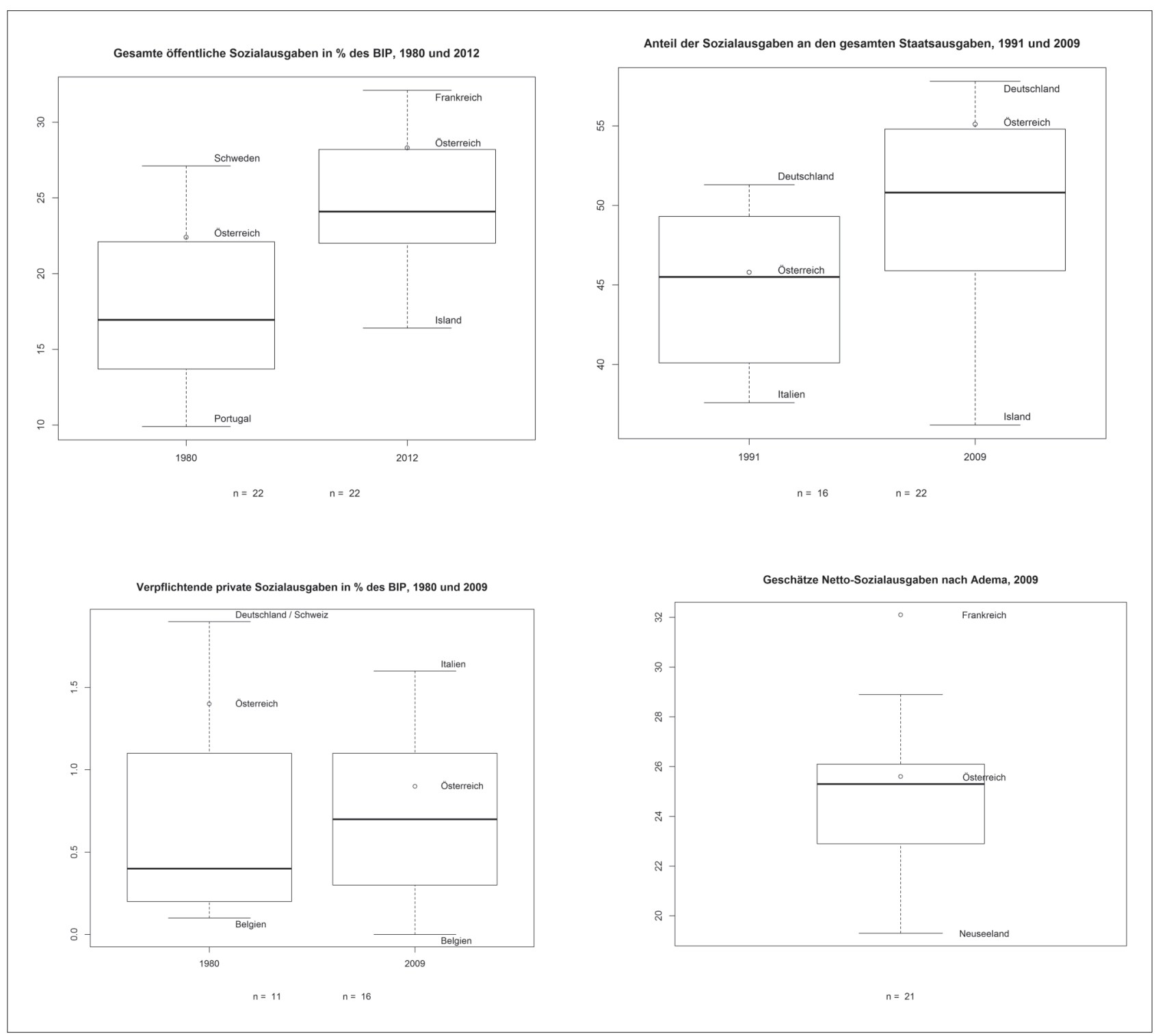

Quelle: OECD Social Expenditure Database

milienausgaben sowie die Ausgaben für passive Arbeitsmarktpolitik entsprechen fast genau dem Medianwert. Überdurchschnittlich hoch sind jedoch die Aufwendungen für familienbezogene Geldleistungen und die aktive Arbeitsmarktpolitik. Hingegen hat Österreich bei den familienbezogenen Dienstleistungen (z.B. Kinderbetreuung) Terrain verloren, sodass heute drei Viertel der Länder mehr Ressourcen für dieses Politikfeld aufwenden.

\section{Finanzierung}

Im Hinblick auf die Finanzierung des Sozialstaates sind zwei Befunde bemerkenswert (Abbildung 3). Zum einen liegt Österreich bei der Steuer- und Abgabenquote im oberen Quartil. In den letzten zehn Jahren ist diese Quote analog zum internationalen Trend leicht gesunken. Unangefochten liegen hier die nordischen Länder an der Spitze, während die Vereinigten Staaten die mit Abstand geringste Steuer- und Abgabenlast aufweisen. Zum anderen nimmt Österreich bei den Sozialversicherungsbeiträgen erwartungsgemäß einen Spitzenplatz ein. Ihr Anteil am BIP hat seit 1980 zugenommen und ist gegenwärtig nur noch in Frankreich höher. Dieser Zuwachs erfolgte in erster Linie bei den Beiträgen der ArbeitnehmerInnen, die im internationalen Vergleich und in Relation zum BIP heute in Deutschland und Österreich am stärksten zur Finanzierung des Sozialstaates beitragen. Demgegenüber liegt die Beitragsbelastung der ArbeitgeberInnen in Österreich nur leicht über dem Median und 
Abbildung 2: Programmspezifische öffentliche Sozialausgaben

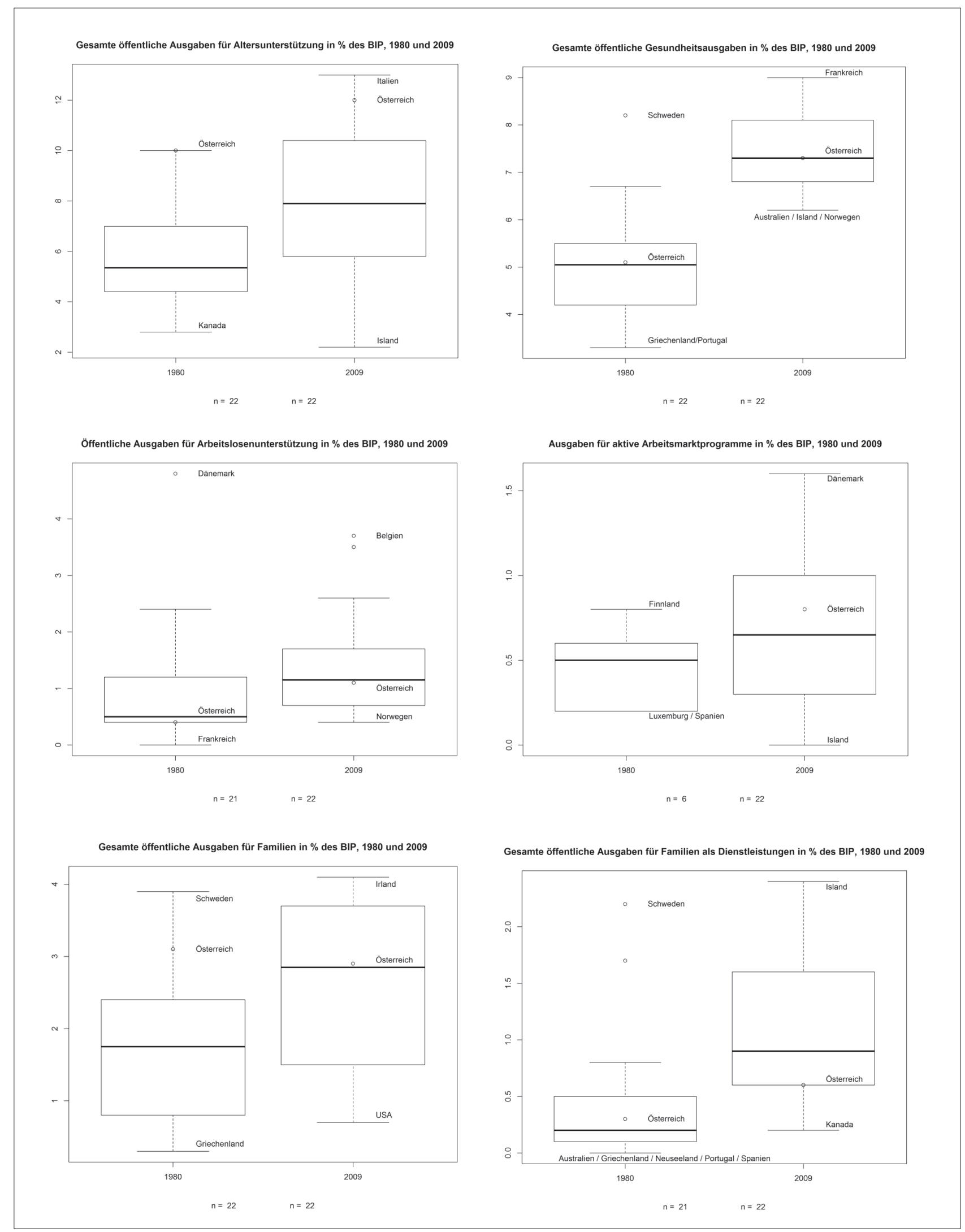


ist zudem über die Zeit relativ konstant geblieben. Die hohe Finanzierungslast der ArbeitnehmerInnen spiegelt sich auch im Steuersystem wider, das den Faktor Arbeit überproportional stark belastet (OECD 20II, 26). Dieser Befund steht nicht zuletzt auch in Zusammenhang mit den Steuerreformen der letzten beiden Jahrzehnte, da bei der Körperschaftssteuer Satzreduktionen vorgenommen wurden, während die Vermögen- und Erbschaftssteuer abgeschafft wurden.

Ein interessantes Bild zeigt sich an der Schnittstelle zwischen Sozialausgaben und Steuersystem, d.h. bezüglich der Frage, ob und inwieweit Transferleistungen besteuert bzw. Eigenvorsorge und betriebliche Sozialleistungen steuerlich gefördert werden. Ein Indikator für das Zusammenspiel zwischen Sozialausgaben und Steu- ersystem ist die Differenz zwischen der Brutto- und der Nettosozialleistungsquote (vgl. Castles/Obinger 2007). Dieses Maß wird durch die Höhe der privaten Sozialausgaben, die steuerliche Förderung individueller und betrieblicher Vorsorgeformen und die Belastung von Sozialtransfers mit Steuern und Sozialabgaben beeinflusst. In Österreich (sowie in den nordischen Ländern) ist die Differenz zwischen den beiden Ausgabenquoten besonders groß, d.h. die öffentliche Bruttosozialleistungsquote ist deutlich höher als die gesamte Nettosozialleistungsquote (vgl. auch Abbildung I). Dies bedeutet, dass entweder Sozialleistungen vergleichsweise stark mit Steuern und Sozialabgaben belastet werden oder/ und Märkte aus der Bereitstellung sozialer Sicherung weit zurückgedrängt werden, was in geringen privaten

Abbildung 3: Steuer- und Abgabenquote sowie Anteile der Sozialversicherungsbeiträge am BIP

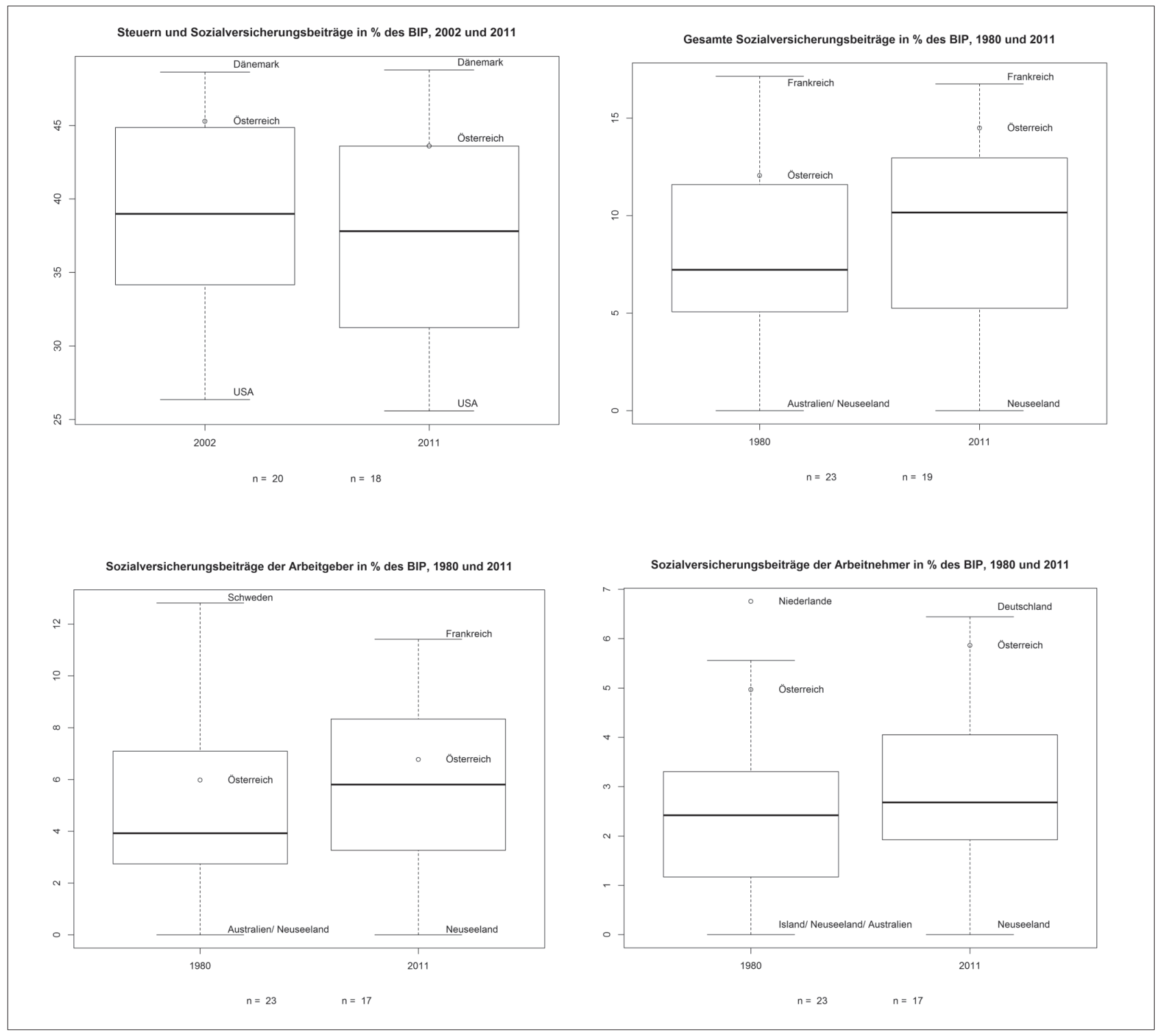

Quelle: OECD Revenue Statistics 
Sozialausgaben bzw. in einer geringen steuerlichen Förderung privater Vorsorgeformen Niederschlag findet. Demgegenüber übersteigen in den meisten englischsprachigen Ländern die Nettosozialausgaben die Bruttoaufwendungen (negative Differenz), sodass dort privater und steuerlich geförderter Sozialpolitik ein hoher Stellenwert zukommt, während soziale Transferleistungen nicht oder nur gering besteuert werden. Abschnitt 4 zeigt, dass daraus erhebliche Konsequenzen für die Einkommensverteilung und Armutsinzidenz resultieren.

\section{Policy Outputs}

Ungeachtet der verbesserten Datenlage sind Sozialausgaben zweifellos "epiphenomenal to the theoretical substance of welfare states" (Esping-Andersen 1990, 19). Kernelemente des Wohlfahrtsstaates sind aus Sicht der skandinavischen Machtressourcentheorie vielmehr „policies to affect outcomes of, and conditions for, distributive processes in the sphere of markets so as to decrease inequality and/or poverty" (Korpi/Palme 2003, 428). Folglich liegt der Fokus der Forschung heute primär auf der Architektur von Wohlfahrtsstaaten und den damit verbundenen Verteilungs- und Beschäftigungswirkungen. Im Folgenden werden die Generosität von Sozialleistungen, die Leistungsart, der Beschäftigungsschutz und der von den sozialen Sicherungssystemen erfasste Personenkreis näher beleuchtet. Die Outcome-Dimension wird dann im nächsten Abschnitt in den Blick genommen.

\section{Leistungsart}

Abbildung 4 stellt die Sozialausgaben nach Leistungstyp dar. Diesbezüglich erweist sich Österreich als ausgesprochen klassischer Bismarckscher Sozialstaat. In keinem anderen OECD-Mitgliedsland ist die soziale Sicherung stärker transferorientiert als in Österreich, wobei der Großteil der Transferausgaben im Pensionssystem gebunden ist. Dementsprechend unterdurchschnittlich sind im internationalen Vergleich die Aufwendungen für Sach- und Dienstleistungen. Österreich ist hier trotz eines Ausgabenzuwachses von der Medianposition in den unteren Quartilbereich zurückgefallen.

\section{Lohnersatzraten und Inklusionsgrad}

Auf Basis der Welfare State Entitlement Database (Scruggs 2013) kann die Generosität der Sozialleistungen im internationalen Vergleich (grob) vermessen werden. Zudem liefert dieser Datensatz Informationen zur personellen Reichweite der Sozialschutzsysteme. Abbildung 5 zeigt die Lohnersatzraten und den Inklusionsgrad (in Relation zur Erwerbsbevölkerung) von drei Sozialschutzprogrammen (Geldleistungen bei Arbeitslosigkeit und im Krankheitsfall sowie die Standardrente). Bei den Lohnersatzraten handelt es sich um die Nettolohnersatzrate für eine alleinstehende Person, wobei der so genannte "Average Production Worker" den Bezugspunkt der Berechnung bildet. Gegenläufig zum Anstieg der Sozialausgaben sind die Leistungsniveaus im internationalen Durchschnitt in zwei von drei Programmen gesunken. Österreich ist insofern ein Ausreißer, als diesen Daten zufolge die Generosität der Sozialleistungen kon-

Abbildung 4: Sozialausgaben nach Leistungsart

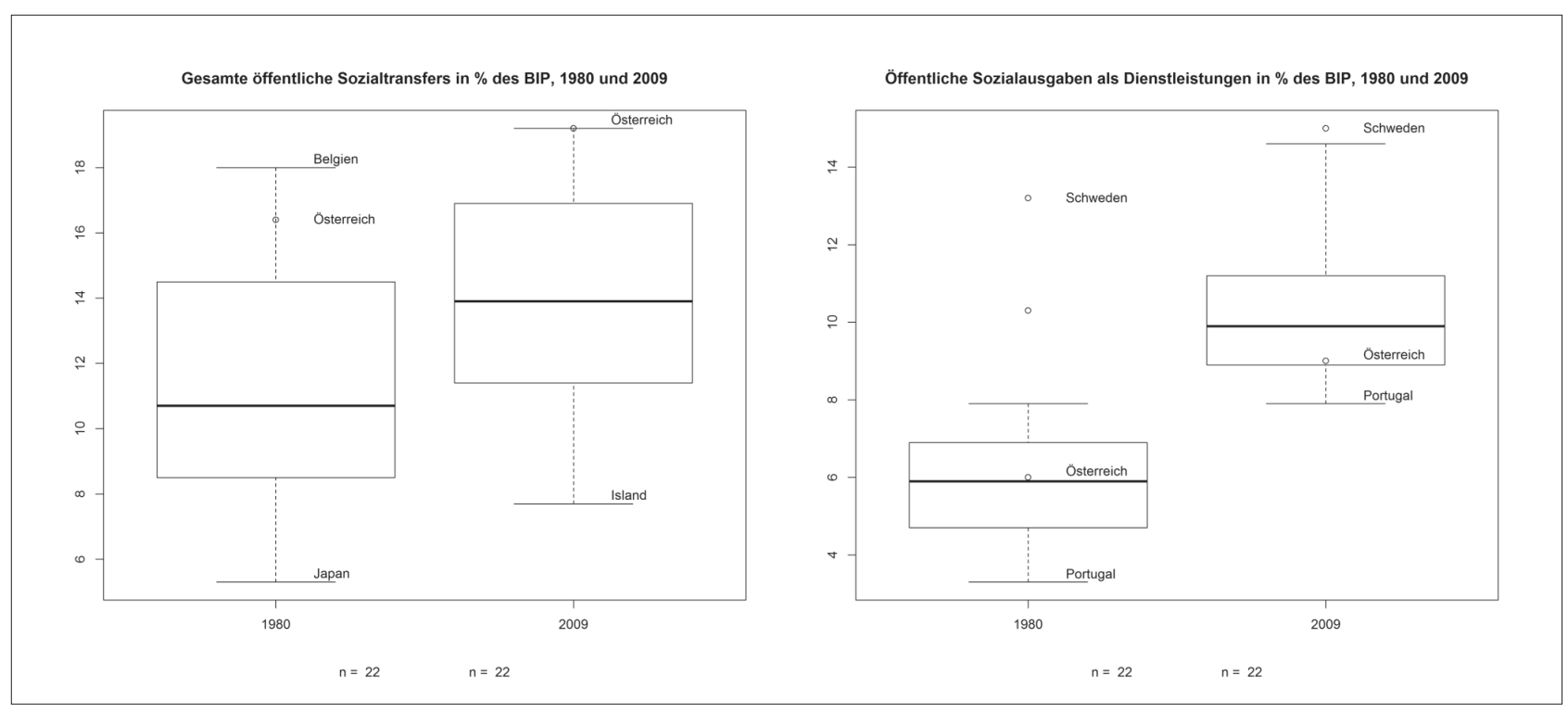

Quelle: OECD Social Expenditure Database 
Abbildung 5: Generosität und Inklusionsgrad von drei Sozialschutzprogrammen (Alleinstehende) in 18 OECD-Ländern

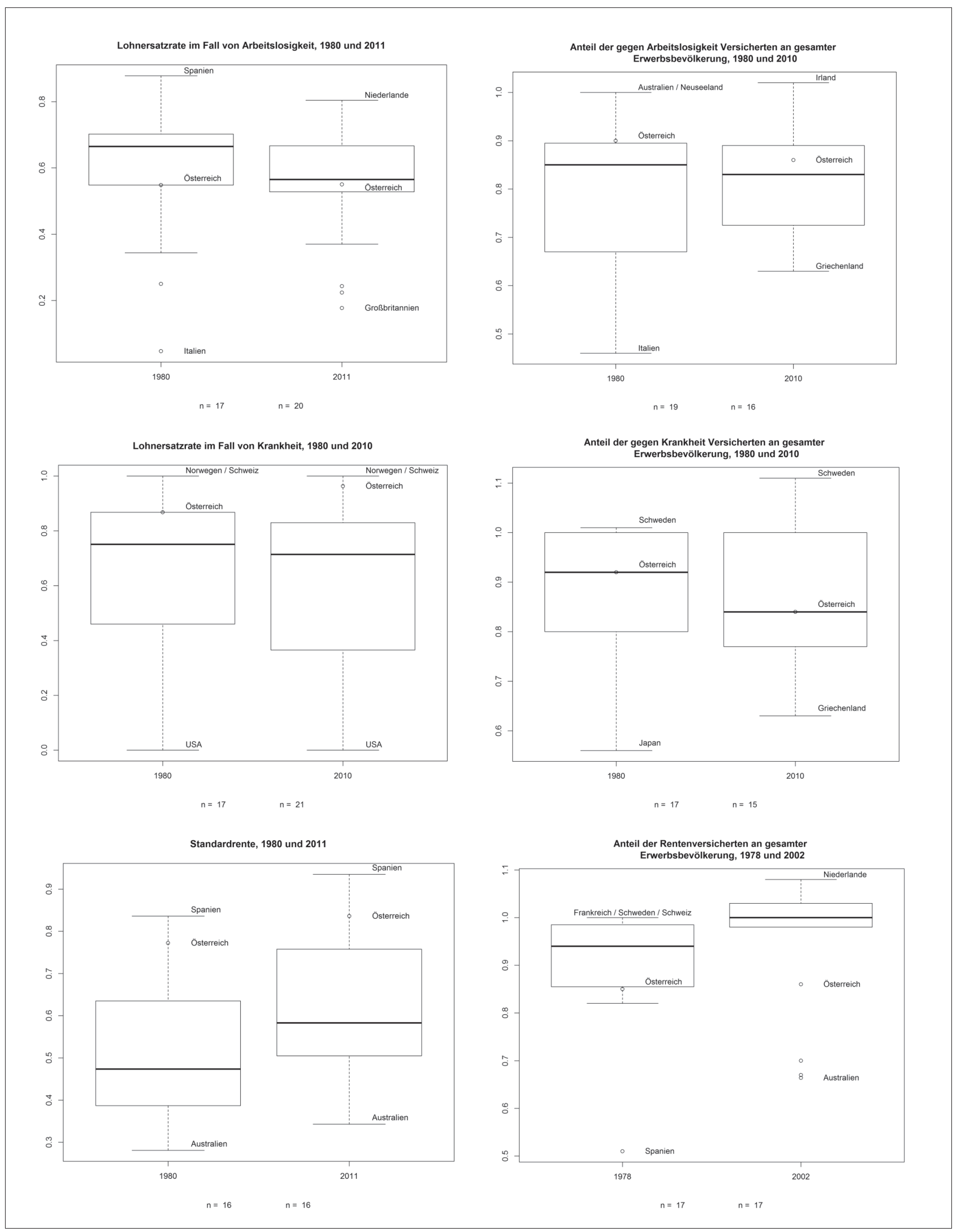

Quelle: Scruggs (2013) 
stant geblieben ist oder sogar zugenommen hat. Im Hinblick auf das Leistungsniveau befindet sich Österreich bei der Arbeitslosenunterstützung im internationalen Mittelfeld, die Geldleistungen im Krankheitsfall und im Bereich der Alterssicherung sind dagegen vergleichsweise hoch. Im Hinblick auf den Inklusionsgrad liegt Österreich zweimal im Mittelfeld, bei der Alterssicherung sogar unterhalb des Medians. Diese Positionierung ist jedoch insofern wenig überraschend, als Sozialversicherungssysteme gegenüber universellen Systemen mit Staatsbürger- oder Wohnsitzprinzip naturgemäß eine geringere personelle Reichweite aufweisen.

Abbildung 6 zeigt zu Informationszwecken für die gleichen Programme eine alternativ berechnete Lohnersatzrate (linke Achse). Für das Arbeits- und Krankengeld bezieht sich der Lohnersatz auf den Mittelwert der Leistung für eine alleinstehende Person und eine vierköpfige Familie mit einem Durchschnittsverdiener. Bei den Pensionsleistungen handelt es sich um den Durchschnitt der Leistung für einen Alleinstehenden und ein Ehepaar. Neben dem OECD-Durchschnitt (dicke schwarze Linie) und dem Variationskoeffizienten (dicke gestrichelte Linie, rechte Achse) sind die Lohnersatzra- ten in drei prototypischen Sozialstaaten dargestellt, wobei die USA den liberalen Sozialstaatstyp, Schweden den sozialdemokratischen Wohlfahrtsstaat und Österreich den kontinentaleuropäischen Sozialversicherungsstaat repräsentiert. Insgesamt entsprechen die Befunde weitgehend jenen in Abbildung 5. Bemerkenswert ist neben den beträchtlichen Leistungskürzungen in Schweden (allerdings ausgehend von einem sehr hohen Leistungsniveau) der Umstand, dass das österreichische Leistungsniveau mit Ausnahme des Arbeitslosengeldes gegenwärtig deutlich über dem OECD-Durchschnitt liegt und sich mittlerweile auch über dem schwedischen Niveau befindet.

\section{Regulierungsdichte des Arbeitsmarktes}

Ein wichtiges, von der vergleichenden Forschung aber stark vernachlässigtes Feld der Sozialpolitik ist die Regulierungsdimension. Entsprechend lückenhaft ist auch die Datenlage. Allerdings stellt die OECD einen Index zur Regulierungsdichte des Arbeitsmarktes zur Verfügung, der einen groben internationalen Quer- und Längsschnittvergleich erlaubt. Abgebildet werden die

Abbildung 6: Nettolohnersatzrate von Arbeitslosengeld, Krankengeld, Mindest- und Standardrenten in 18 OECD Ländern (im Uhrzeigersinn von oben links), 1971-2011

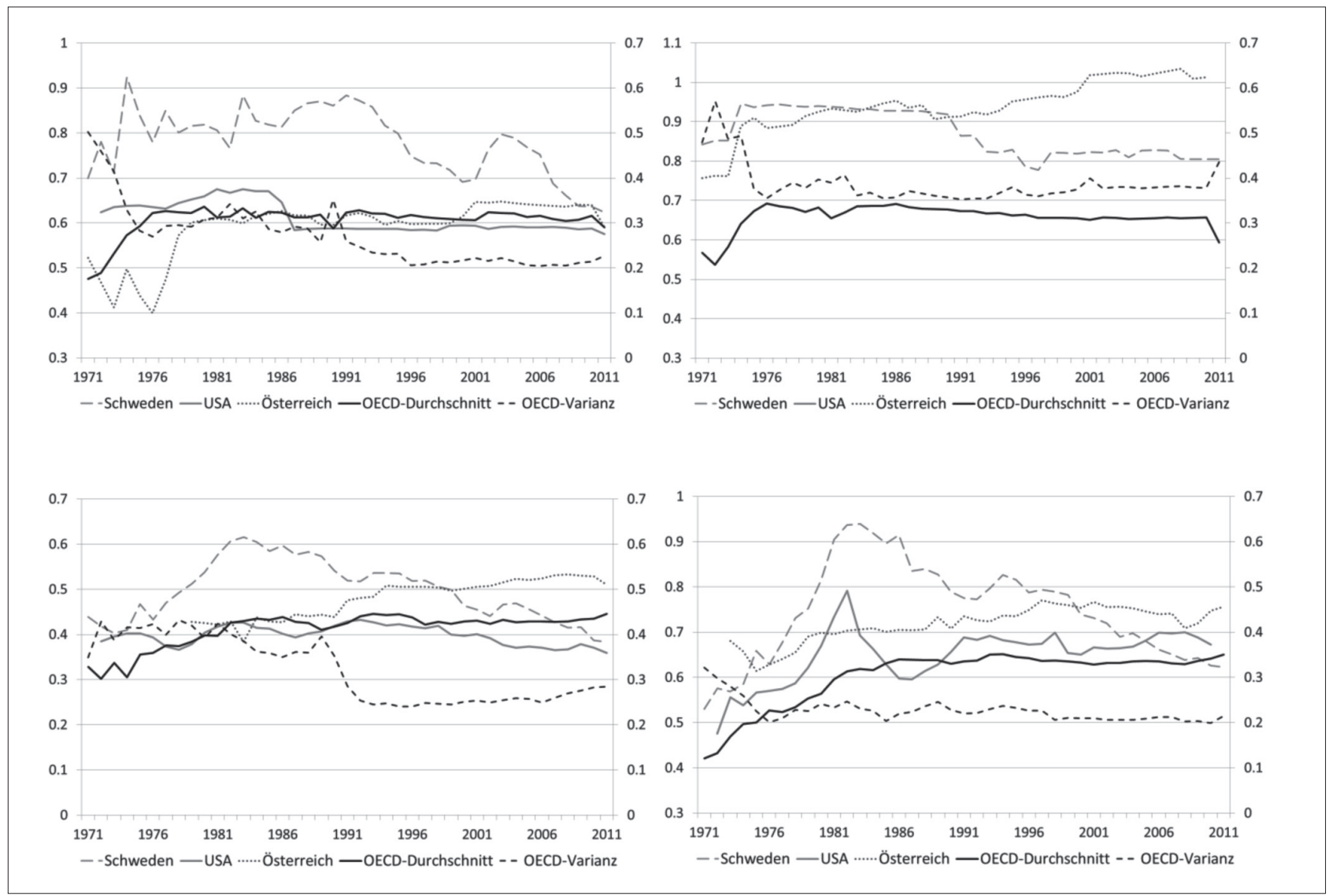


Abbildung 7: Regulierungsdichte des Arbeitsmarktes

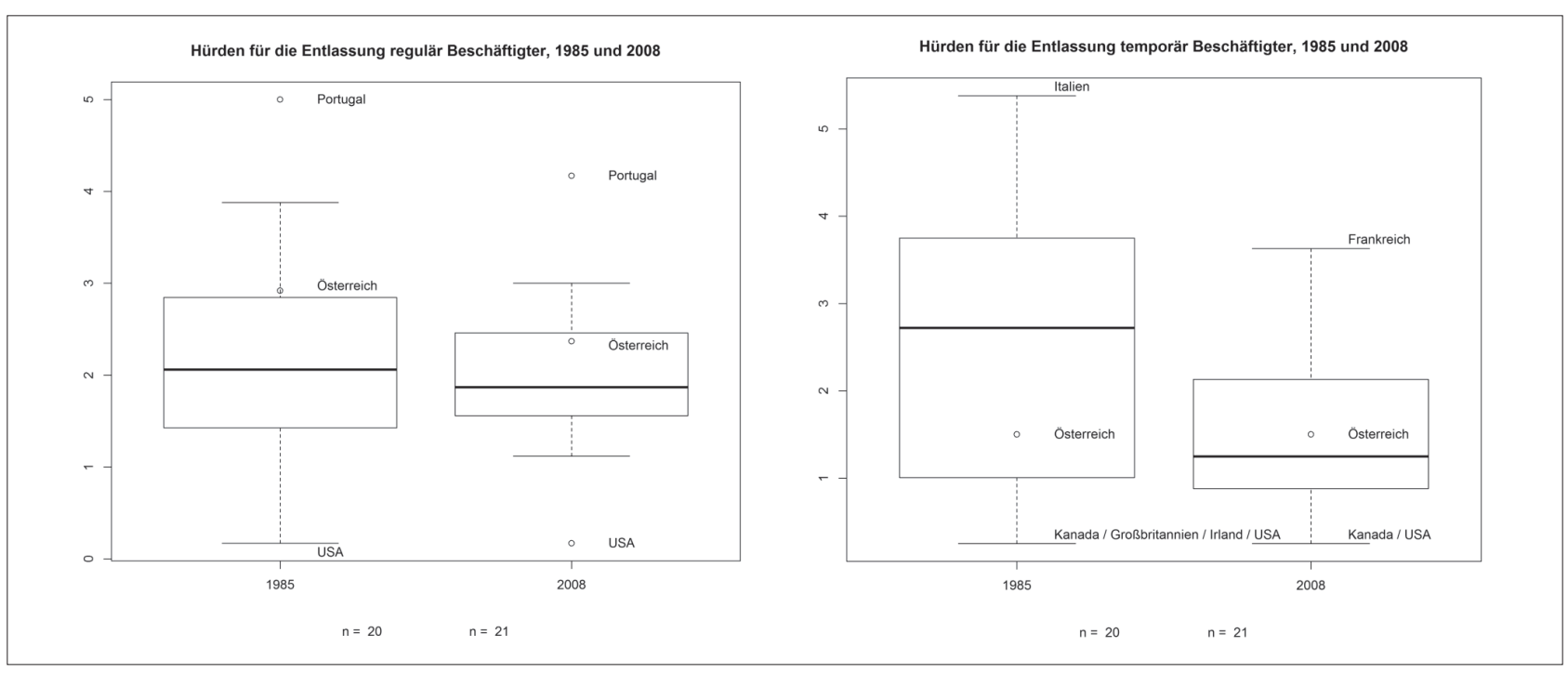

Quelle: OECD Indicators on Employment Protection

rechtlichen Kündigungshürden, wobei zwischen regulär und temporär Beschäftigten unterschieden wird. Dieser Index kann Werte zwischen null und sechs annehmen. Hohe Werte spiegeln einen ausgeprägten Beschäftigungsschutz wider, während niedrige Werte geringe Schutzbestimmungen anzeigen. Abbildung 7 stellt diesen Index für beide Beschäftigungsgruppen dar. Bei den temporär Beschäftigten ist im internationalen Vergleich eine deutliche Deregulierung im Zeitverlauf festzustellen, während bei den regulär Beschäftigten kaum Veränderungen stattgefunden haben. Bei den befristet Beschäftigten deutet die Stauchung der Box auf einen starken Konvergenzprozess hin. Der Arbeitsmarkt wurde also primär an den Rändern dereguliert, was als Indiz für eine zunehmende Dualisierung zwischen gut geschützten InsiderInnen und den als Flexibilitätsreserve fungierenden atypisch Beschäftigten gesehen wird (Emmenegger et al. 20I2). Diese Diskrepanz zwischen InsiderInnen und OutsiderInnen ist auch für Österreich zu beobachten, allerdings weicht Österreich in zweifacher Hinsicht vom internationalen Trend ab. Einerseits wurde diesen Daten zufolge der Beschäftigungsschutz der Insider leicht gelockert, andererseits war der Beschäftigungsschutz für befristete Arbeitsverhältnisse immer schon sehr gering und blieb über die Zeit auch unverändert.

\section{Outcomes}

Welche Effekte und Auswirkungen resultieren aus diesem Sozialstaatsprofil und wie schneidet Österreich im internationalen Vergleich ab? Zentrale sozialstaatliche Outcomeindikatoren sind gemäß der Machtressourcen- theorie die sekundäre Einkommensverteilung, die Armutsinzidenz und die Arbeitsmarktperformanz (Korpi/ Palme 2003), zumal diese Größen maßgeblich über individuelle Aufstiegs- und Teilhabechancen entscheiden. Allerdings ist $\mathrm{zu}$ beachten, dass diese Performanzindikatoren neben der Sozialpolitik noch von zahlreichen anderen Faktoren (z.B. Steuerpolitik, Bildungsniveau, Sozialstruktur) beeinflusst werden.

\section{Armutsbekämpfung und Einkommensverteilung}

Abbildung 8 zeigt die Entwicklung der Armutsquote und der Einkommensverteilung vor und nach Steuern und Transfers sowie die Einkommensverteilung innerhalb der Gruppe der über 65-Jährigen. Die Armutsquote reflektiert den Anteil der Personen mit einem Einkommen von weniger als der Hälfte des Medianeinkommens. Die Einkommensverteilung wird durch den Ginikoeffizienten abgebildet, wobei hohe Werte dieses Konzentrationsmaßes auf eine hohe Ungleichheit hinweisen. Alle Boxplots deuten eindeutig auf einen Anstieg von Armut und Einkommensungleichheit in der OECD-Welt hin. Insbesondere in Bezug auf die Einkommensverteilung ist die hohe Umverteilungswirkung des österreichischen Steuer- und Transfersystems bemerkenswert. Während die primäre Einkommensverteilung sehr ungleich ist und Österreich hier über dem Durchschnitt liegt, rutscht Österreich nach Berücksichtigung von Steuern und Transfers auf eine Position im unteren Quartil. Ähnliches gilt auch für die Einkommensverteilung bei den über 65-Jährigen. Auch die Armutsquote ist trotz eines Anstiegs in den letzten Jahren vergleichsweise gering. Ähnlich gute bzw. noch bessere Erfolge bei der Eindämmung von Ungleichheit und Armut verbuchen nur alle 
Abbildung 8: Armuts- und Verteilungsindikatoren

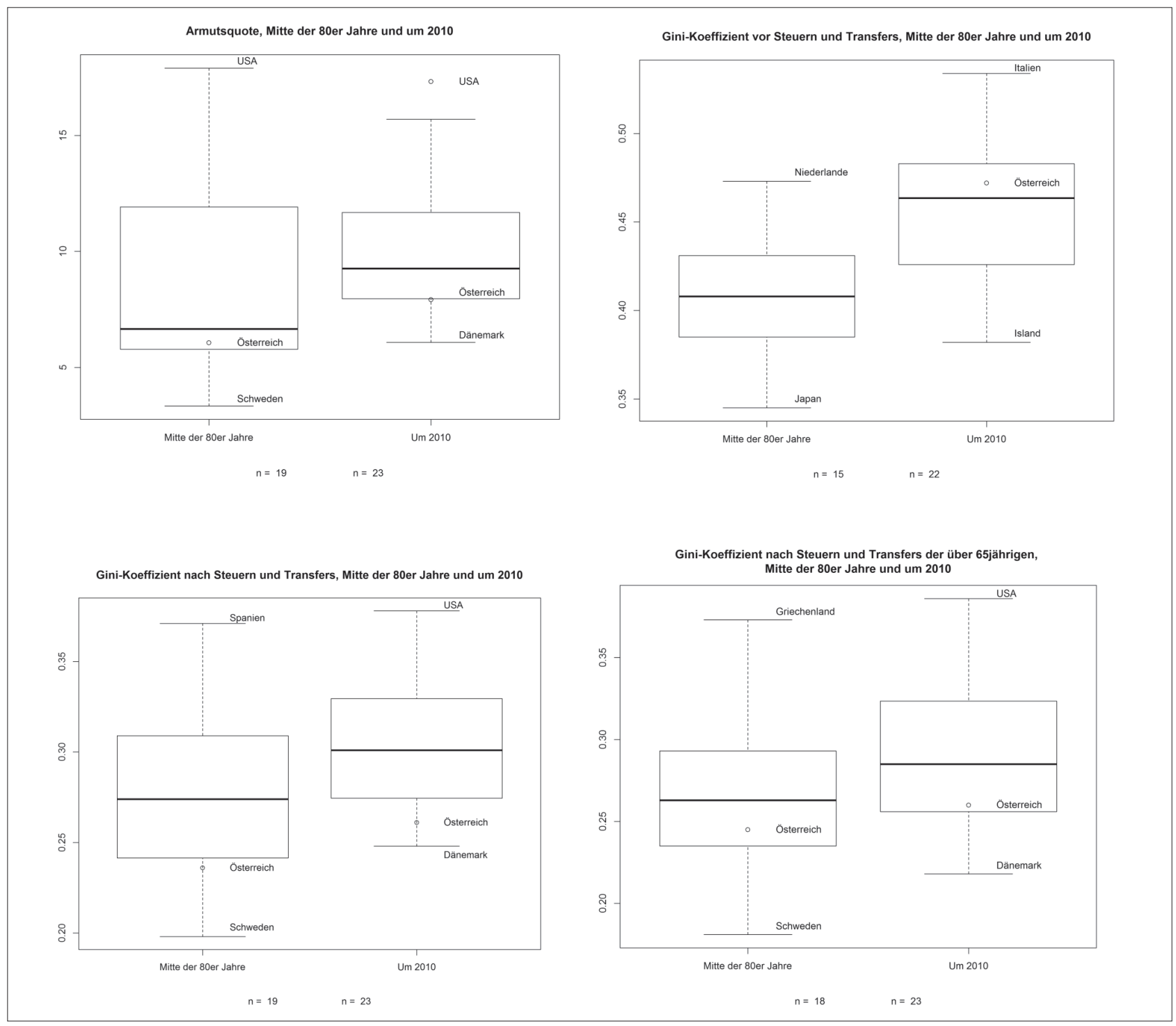

Quelle: OECD Income Distribution Database

nordischen Staaten ${ }^{7}$, während die Vereinigten Staaten und die krisengebeutelten südeuropäischen Ländern hier große Defizite besitzen.

\section{Beschäftigung und Arbeitsmarkt}

Abbildung 9 zeigt verschiedene beschäftigungspolitische Performanzindikatoren vor und nach der jüngsten weltweiten Wirtschaftskrise. Trotz der Weltwirtschaftskrise blieben die Arbeitslosenquoten sowohl für Männer

7 Wie bereits erwähnt ist in den nordeuropäischen Sozialstaaten und in Österreich der Stellenwert steuerlich geförderter privater Vorsorgeformen, die v.a. mittlere und hohe Einkommen begünstigen, relativ gering. Zusammen mit der höheren Besteuerung von Sozialtransfers, die einen zusätzlichen Umverteilungsmechanismus darstellt, dürfte dies zu einer vergleichsweise günstigen sekundären Einkommensverteilung beitragen. als auch Frauen vergleichsweise niedrig. In beiden Fällen liegt Österreich im unteren Quartil. Besonders erfolgreich ist Österreich (wie die übrigen deutschsprachigen Länder) bei der Bekämpfung der Jugendarbeitslosigkeit, was wesentlich der dualen Berufsbildung geschuldet ist. Diesbezüglich schneidet Österreich deutlich besser ab als die nordischen Sozialstaaten. Unterdurchschnittlich ist auch die Arbeitslosigkeit innerhalb der Gruppe der 55-64-Jährigen, wenngleich hier zu berücksichtigen ist, dass die Erwerbsbeteiligung dieser Altersgruppe in Österreich aufgrund eines niedrigen Renteneintrittsalters vergleichsweise sehr niedrig ist. Bemerkenswert ist, dass die Beschäftigungsquote trotz Wirtschaftskrise und gegenläufig zum internationalen Trend angestiegen und heute leicht überdurchschnittlich ist. Auch die Frauenerwerbsbeteiligung ist höher als in fast allen liberalen und 
Abbildung 9: Beschäftigung und Arbeitslosigkeit

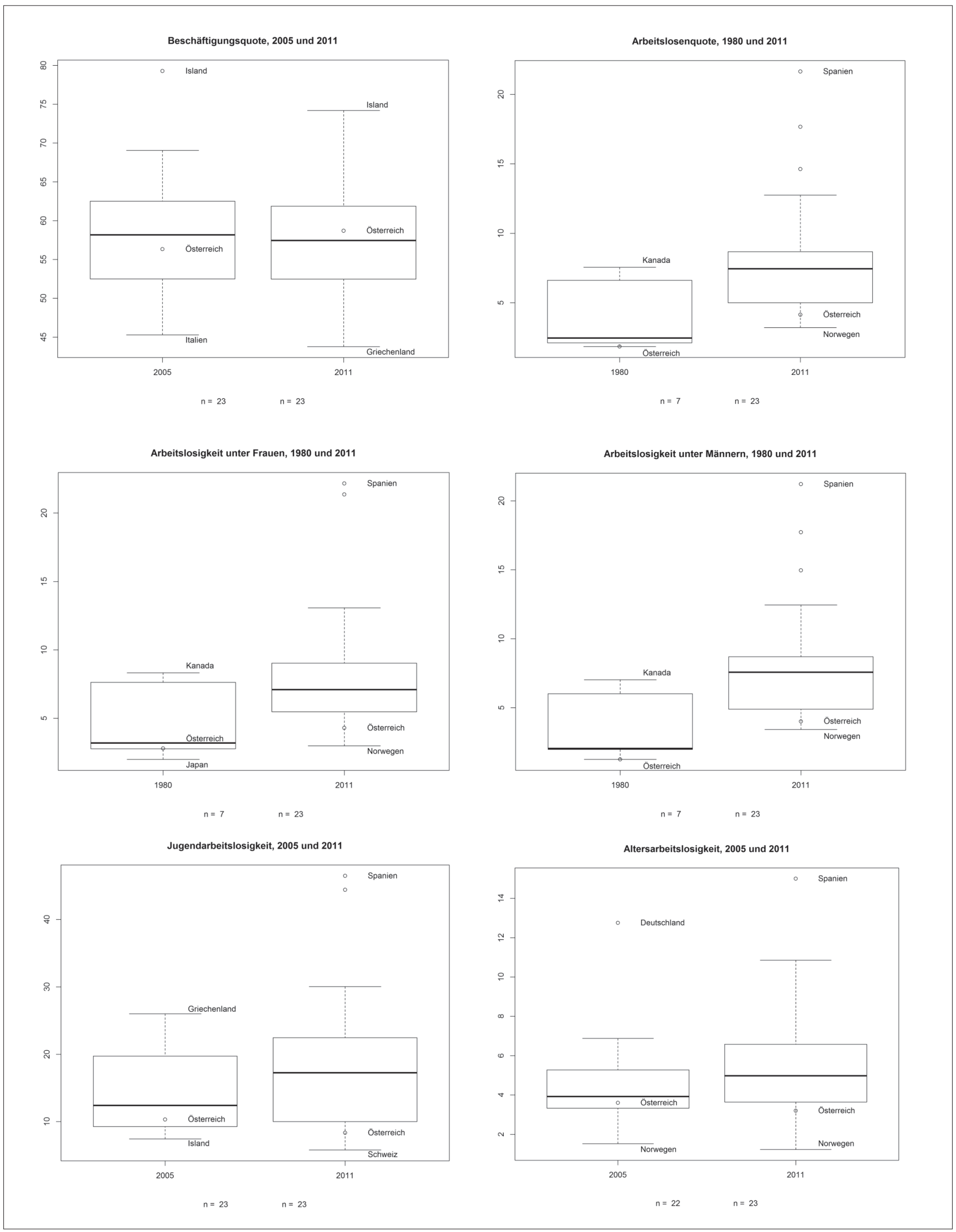


kontinentalen Ländern und reicht fast an das Niveau der nordischen Länder heran ${ }^{8}$. Während in Österreich die Krise insgesamt vergleichsweise glimpflich verlief, führte die Wirtschaftskrise auf den Arbeitsmärkten der südeuropäischen Länder zu dramatischen sozialen Verwerfungen und trieb die Arbeitslosenquoten, insbesondere jene der Jugendlichen, in astronomische Höhen.

\section{Zusammenfassung und Diskussion}

Welche Schlussfolgerung lässt sich nun in der Zusammenschau von 33 Makroindikatoren im Hinblick auf die Position von Österreichs Sozialstaat im internationalen Vergleich ziehen? Tabelle I gibt, differenziert nach den drei Analysedimensionen, darüber Auskunft. extrem hohe Transferorientierung auf der Leistungsebene, den hohen Anteil der Sozialversicherungsbeiträge auf der Finanzierungsseite, das hohe Ausgabenniveau für die Alterssicherung und den hohen Anteil der öffentlichen Sozialausgaben an den Gesamtausgaben des Staates, der auf der Einnahmenseite durch eine vergleichsweise hohe Steuer- und Abgabenquote gespiegelt wird. Bemerkenswert ist vor allem, dass trotz markanter konservativer Strukturmerkmale die Leistungsfähigkeit des Sozialstaates überdurchschnittlich hoch ist. Sowohl bei der Bekämpfung von Armut und Einkommensungleichheit als auch bei der Beschäftigungsperformanz befindet sich Österreich fast durchwegs im internationalen Spitzenfeld und ist den nordischen Ländern fast ebenbürtig. Gleiches gilt mittlerweile auch für die Generosität der Sozialleistungen. Bei den übrigen Output-

Tabelle 1: Die Position Österreichs im OECD-Vergleich ca. im Jahr 2010

\begin{tabular}{|c|c|c|c|c|c|c|c|c|c|}
\hline & \multicolumn{2}{|c|}{$\begin{array}{c}\text { INPUT } \\
\text { (14 Indikatoren) }\end{array}$} & \multicolumn{3}{|c|}{$\begin{array}{c}\text { OUTPUT } \\
\text { (10 Indikatoren) }\end{array}$} & \multicolumn{3}{|c|}{$\begin{array}{c}\text { OUTCOME } \\
\text { (9 Indikatoren) }\end{array}$} & \multirow{2}{*}{$\begin{array}{l}\text { TOTAL } \\
\mathrm{N}=33\end{array}$} \\
\hline & $\begin{array}{l}\text { Ausgaben } \\
(\mathrm{N}=10)\end{array}$ & $\begin{array}{c}\text { Finanzie- } \\
\text { rung } \\
(\mathrm{N}=4)\end{array}$ & $\begin{array}{l}\text { Leis- } \\
\text { tungsart } \\
(\mathrm{N}=2)\end{array}$ & $\begin{array}{c}\text { Genero- } \\
\text { sität } \\
(\mathrm{N}=3)\end{array}$ & $\begin{array}{l}\text { Inklusion } \\
\qquad(\mathrm{N}=3)\end{array}$ & $\begin{array}{c}\text { Regulie- } \\
\text { rung } \\
(\mathrm{N}=2)\end{array}$ & $\begin{array}{c}\text { Vertei- } \\
\text { lung } \\
(\mathrm{N}=3)\end{array}$ & $\begin{array}{c}\text { Arbeits- } \\
\text { markt } \\
(\mathrm{N}=6)\end{array}$ & \\
\hline Ausreißer nach unten & 0 & 0 & 0 & 0 & 1 & 0 & 0 & 0 & 1 \\
\hline Minimum & 0 & 0 & 0 & 0 & 0 & 0 & 0 & 0 & 0 \\
\hline Unteres Quartil & 0 & 0 & 0 & 0 & 0 & 0 & 2 & 5 & 7 \\
\hline Interquartilsbereich & 7 & 1 & 1 & 1 & 2 & 2 & 1 & 1 & 16 \\
\hline Oberes Quartil & 3 & 3 & 0 & 2 & 0 & 0 & 0 & 0 & 8 \\
\hline Maximum & 0 & 0 & 1 & 0 & 0 & 0 & 0 & 0 & 1 \\
\hline Ausreißer nach oben & 0 & 0 & 0 & 0 & 0 & 0 & 0 & 0 & 0 \\
\hline
\end{tabular}

Bei weniger der Hälfte (I6 von 33) der Indikatoren liegt Österreich innerhalb des Interquartilbereichs, d.h. im Bereich der mittleren 50\% der Verteilung. Dies passt nicht unbedingt zu einem mittleren sozialpolitischen Weg, den man kontinentaleuropäischen Ländern gemeinhin zuschreibt. In acht Fällen befindet sich Österreich im oberen Quartil und in sieben Fällen im unteren Quartil. Nur jeweils einmal nimmt Österreich einen Spitzenplatz bzw. eine Ausreißerplatzierung ein. Betrachtet man die I7 Abweichungen vom Interquartilbereich genauer, zeigt sich ein interessantes Bild. Die Abweichungen nach oben spiegeln allesamt klassische Bismarcksche Strukturmerkmale wider. Dies betrifft die

8 Die Beschäftigungsquote von Frauen betrug 2012 nach Angaben der OECD Labour Market Statistics Database 67,9\% (zum Vergleich: Finnland: 68,2\%; Dänemark: 70\%; Schweden: 71,8\%). Zu beachten ist aber, dass in den nordischen Ländern mehr Frauen vollzeiterwerbstätig sind, während in Österreich die Teilzeitquote deutlich höher ist. indikatoren belegt Österreich hingegen einen Platz im Mittelfeld. Der vergleichsweise geringe Inklusionsgrad in der Alterssicherung stellt den einzigen Ausreißer nach unten dar.

In der Zusammenschau aller drei Dimensionen lässt sich somit festhalten, dass ein überdurchschnittlich hoher öffentlicher Ressourcenaufwand und ausgeprägte Bismarcksche Strukturelemente des Sozialstaates mit einer vergleichsweise guten Leistungsperformanz korrespondieren. Dies kann nicht nur als ein Indiz für eine beachtliche Effektivität des österreichischen Sozialstaates gewertet werden, sondern der Makrovergleich zeigt auch, dass Bismarcksche Sozialstaaten keinesfalls zwangsläufig eine inferiore Leistungsbilanz aufweisen?. Da gerade innerhalb der kontinentalen Wohlfahrtsstaaten die Varianz hoch ist (Lessenich/Ostner 1998), sind

\footnotetext{
9 Lohnenswert für künftige Forschungen wäre eine tiefenschärfere Aufschlüsselung der Output- und Outcomeindikatoren nach Merkmalen wie Geschlecht, Staatsbürgerschaft, Bildungsgrad oder Alter.
} 
voreilige Rückschlüsse von der Typenzugehörigkeit auf die Performanz von Sozialstaaten unangebracht ${ }^{10}$.

All das heißt nicht, dass es keine Problemzonen und Reformbedarfe gibt. Schwächen offenbaren sich insbesondere dann, wenn die österreichische Sozialstaatsarchitektur auf ihre Nachhaltigkeit bzw. Zukunftsfestigkeit überprüft wird. Zwei Problemzonen sind besonders virulent. Erstens ist die soziale Sicherung heute ausgesprochen transferorientiert, während die finanziellen Aufwendungen für Sach- und Dienstleistungen, insbesondere für familienbezogene soziale Dienstleistungen, gering sind. Diesen kommt aber gerade für die sozialpolitische Bewältigung der so genannten neuen sozialen Risiken (vgl. Fink 2009) eine Schlüsselrolle zu. Von den sehr hohen Transferausgaben profitieren überproportional die Älteren. Ca. I4\% des BIP werden für die Alters- und Hinterbliebenenversorgung aufgewendet, während der OECD-Durchschnitt nur 9,6\% beträgt. Ein Grund für die weit überdurchschnittlich hohen Ausgaben für die Alterssicherung ist unter anderem das niedrige faktische Pensionsantrittsalter bzw. die im internationalen Vergleich sehr geringe Erwerbsbeteiligung der 55-64-Jährigen, wodurch Arbeitsmarktprobleme nicht nur in das Alterssicherungssystem verlagert, sondern auch kaschiert werden.

Eine zweite Problemzone wird erst sichtbar, wenn mit der Bildungspolitik ein Politikfeld berücksichtigt wird, das im englischsprachigen Raum traditionell als Teil des Wohlfahrtsstaates gesehen wird. Die heimischen Bildungsausgaben liegen heute unter dem Durchschnitt vergleichbarer Länder (OECD 20I3). In fiskalischer Hinsicht klafft folglich eine sehr große Lücke zwischen den Ausgaben für Bildung und Alterssicherung, die nur noch in Griechenland und Italien größer ist. Hinzu kommt eine von den PISA-Studien dokumentierte geringe soziale Durchlässigkeit des Bildungssystems sowie dessen vergleichsweise mediokre Effektivität bei der Vermittlung elementarer schulischer Kompetenzen. Der österreichische Sozialstaat ist insgesamt also vergleichsweise stark vergangenheitsbezogen und zu wenig zukunftsorientiert ausgerichtet. Der Wohlfahrtsstaat des 2I. Jahrhunderts benötigt angesichts veränderter sozialer Risiken neben armutsfesten Transfersystemen jedoch auch hohe Bildungsinvestitionen sowie ein breites Angebot an sozialen Dienstleistungen.

IO $\mathrm{Zu}$ den politischen Ursachen für die vergleichsweise günstige und den nordischen Sozialstaaten ähnelnde sozialpolitische Performanz zählen m.E. die Sozialpartnerschaft bzw. die jahrzehntelange Regierungsbeteiligung einer sozialdemokratischen Partei in der Nachkriegszeit. Diesbezüglich unterscheidet sich Österreich grundlegend von anderen Bismarckstaaten.

\section{Literatur}

Adema, Willem/Pauline Fron/Maxime Ladaique (2OII). Is the European Welfare State Really More Expensive? OECD Social, Employment and Migration Working Papers No I24, Paris.

Alber, Jens (1998). Der deutsche Sozialstaat im Licht international vergleichender Daten, in: Leviathan, Vol. 26(2), 199-227.

Castles, Francis G./Herbert Obinger (2007). Social Expenditure and the Politics of Redistribution, in: Journal of European Social Policy, Vol. I7(3), 206-222.

Castles, Francis G. (2009). What Welfare States do: a disaggregated expenditure approach, in: Journal of Social Policy, Vol. 38(I), 45-62.

Emmenegger, Patrick/Silja Häusermann/Bruno Palier/ Martin Seeleib-Kaiser (Hg.) (2012). The Age of Dualization. The Changing Face of Inequality in Deindustrializing Societies, Oxford.

Esping-Andersen, Gøsta (1990). The Three Worlds of Welfare Capitalism, Oxford.

Esping-Andersen, Gøsta (Hg.) (1996). Welfare States in Transition. National Adaptations in Global Economies, London.

Esping-Andersen, Gøsta/Duncan Gallie/Anton Hemerijck/ John Myles (2002). Why We Need a New Welfare State, Oxford.

Fink, Marcel (2009). Neue soziale Risiken (NSR) in Österreich: die aktuelle Wirtschaftskrise als Window of Opportunity zur bedarfsgerechten Reform tradierter Sozialstaatlichkeit?, in: WISO: Wirtschafts- und sozialpolitische Zeitschrift, Vol. 32(3), 95-II3.

Gilbert, Neil (2002). Transformation of the Welfare State: The Silent Surrender of Public Responsibility, Oxford.

Heitzmann, Karin/August Österle (2008). Lange Traditionen und neue Herausforderungen: Das österreichische Wohlfahrtssystem, in: Klaus Schubert/Simon Hegelich/Ursual Bazant (Hg.): Europäische Wohlfahrtssysteme: Ein Handbuch, Wiesbaden, 47-69.

Hemerijck, Anton (2013). Changing Welfare States, Oxford.

Korpi, Walter/Joakim Palme (2003). New Politics and Class Politics in the Context of Austerity and Globalization: Welfare State Regress in I8 Countries, 19751997, in: American Political Science Review, Vol. 97, 425-446.

Lessenich, Stephan/Ilona Ostner (Hg.) (1998). Welten des Wohlfahrtskapitalismus. Der Sozialstaat in vergleichender Perspektive, Frankfurt a.M.

OECD (201I). OECD Economic Surveys. Austria, Paris.

OECD (2013). Education at a Glance 2013. OECD Indicators, Paris. 
Obinger, Herbert/Uwe Wagschal (20I0). Social Expenditure and Revenues, in: Francis G. Castles/Stephan Leibfried/Jane Lewis/Herbert Obinger/Christopher Pierson (Hg.): The Oxford Handbook of the Welfare State, Oxford, 333-352.

Obinger, Herbert/Emmerich Tálos (2010). Janus-faced Developments in a Prototypical Bismarckian Welfare State. Welfare Reforms in Austria Since the I970s, in: Bruno Palier (Hg.), Palier, Bruno (Hg.): A Long Goodbye to Bismarck? The Politics of Welfare Reforms in Continental Europe, Amsterdam, IOI-I28.

Obinger, Herbert (2014). Deutschland im Vergleich zentraler Sozialstaatsindikatoren, in: Peter Masuch/ Wolfgang Spellbrink/Ulrich Becker/Stephan Leibfried (Hg.): Grundlagen und Herausforderungen des Sozialstaats. Denkschrift 6o Jahre Bundessozialgericht, Heidelberg, 47-70.

Obinger, Herbert/Peter Starke (2015). Welfare State Transformation: Convergence and the Rise of the SupplySide Model, in: Stephan Leibfried/Evelyne Huber/ Matthew Lange/Jonah D. Levy/Frank Nullmeier/John D. Stephens (Hg.): The Oxford Handbook of Transformations of the State, Oxford, i.E.

Palier, Bruno (Hg.) (2010). A Long Goodbye to Bismarck? The Politics of Welfare Reforms in Continental Europe, Amsterdam.

Palier, Bruno/Kathleen Thelen (2010). Institutionalizing Dualism: Complementarities and Change in France and Germany, in: Politics \& Society, Vol. 38, II9-I48.

Schmidt, Manfred G.(2005). Sozialpolitik in Deutschland. Historische Entwicklung und internationaler Vergleich, Wiesbaden.

Schmidt, Manfred G. (2012). Wirklich nur Mittelmaß? Deutschlands Sozialstaat im Spiegel neuer, international vergleichender Daten, in: Zeitschrift für Staats- und Europawissenschaften, 2012/2, 159-195.

Scruggs, Lyle (2013). Welfare State Entitlements Data Set: A Comparative Institutional Analysis of Eighteen Welfare States, Version 2.0. Storrs, CT: University of Connecticut.

Tálos, Emmerich (2005). Vom Siegeszug zum Rückzug. Sozialstaat Österreich 1945-2005, Innsbruck.

Tálos, Emmerich (2013). Bestimmungsfaktoren der Sozialpolitik. Am Beispiel des österreichischen Sozialstaates, in: Klaus Armingeon (Hg.): Staatstätigkeiten, Parteien und Demokratie. Festschrift für Manfred G. Schmidt, Wiesbaden, 33I-352.

Unger, Brigitte/Karin Heitzmann (2003). The Adjustment Path of the Austrian Welfare State: back to Bismarck?, in: Journal of European Social Policy, Vol. I3 (4), 37I-387.

\section{Autor}

Herbert Obinger ist Professor für vergleichende Staatstätigkeitsforschung und Sozialpolitik an der Universität Bremen. Forschungsschwerpunkte: Vergleichende Wohlfahrtsstaatsforschung, Politische Ökonomie 